\title{
Photoreaction of neutral 9-aminoacridine in glycerol:water glass characterized by satellite holes and antiholes
}

\author{
Chien-Chih Chiang ${ }^{\text {a,b }}$, Chung-Yuan Mou ${ }^{\mathrm{b}}$, Ta-Chau Chang ${ }^{\mathrm{a}, *}$ \\ ${ }^{a}$ Institute of Atomic and Molecular Sciences, Academia Sinica, P.O. Box 23-166, Taipei 10764, Taiwan, ROC \\ ${ }^{\mathrm{b}}$ Department of Chemistry, National Taiwan University, Taipei, Taiwan, ROC
}

Received 3 March 1997; in final form 15 April 1997

\begin{abstract}
Satellite holes and antiholes in the hole burned (HB) spectrum are used to determine the photoproduct of 9-aminoacridine (9AA) doped in glycerol:water $\left(\mathrm{Gl}: \mathrm{H}_{2} \mathrm{O}\right)$ glass with $\mathrm{pH}=13$. Although monoprotonated 9AA is the photoproduct of the imino form of neutral 9AA, antihole recovery implies that the excited state 9AA returns to original form of 9AA predominantly. Furthermore, similar satellite holes but different antihole structures are observed in the HB spectrum of 9AA doped in polyvinyl butyral film, implying that different HB mechanisms are involved. (O) 1997 Elsevier Science B.V.
\end{abstract}

\section{Introduction}

Photo-induced reaction of chromophores doped in amorphous glasses can deplete the population at the original site and produce a persistent hole in the absorption spectrum at low temperature [1-5]. When tuning the excitation to the vibronic absorption band, several satellite holes resulting from Franck-Condon (FC) active vibrational modes can be produced in the hole burned (HB) spectrum [6-10]. Recently, the high resolution of satellite holes have been used to characterize the tautomeric structures of 9aminoacridine (9AA) doped in glycerol:water $\left(\mathrm{Gl}: \mathrm{H}_{2} \mathrm{O}\right)$ glasses at various $\mathrm{pH}$ values [10]. Specifically, the 514 and $1368 \mathrm{~cm}^{-1}$ satellite holes can be used to characterize the monoprotonated 9AA, while the absence of the $514 \mathrm{~cm}^{-1}$ satellite hole and the

\footnotetext{
${ }^{*}$ Corresponding author
}

frequency shift from $1368 \mathrm{~cm}^{-1}$ to the $1338 \mathrm{~cm}^{-1}$ satellite hole can be used to identify the imino form of neutral 9AA. Furthermore, stronger couplings among the 390,1162 and $1338 \mathrm{~cm}^{-1}$ modes were present in the combination modes in the imino form of neutral 9AA, but more fundamental modes, for instance, the 514, 650 and $690 \mathrm{~cm}^{-1}$ modes, were observed for monoprotonated 9AA. In addition, the coincidence between the antiholes in the spectrum of neutral 9AA and the absorption bands of monoprotonated 9AA suggests that monoprotonated 9AA is the dominant photoproduct of the neutral 9AA. The antihole in the HB spectrum has been used to characterize the mechanism of the photochemical $\mathrm{HB}$ of chlorin [11].

Very recently, Chiang et al. [12] have confirmed that proton transfer between the chromophores and the matrix upon electronic excitation is the main mechanism for the hole-burning of the protonated acridine derivatives doped in polyvinyl alcohol (PVOH) films. Although we proposed that excited 
state proton transfer between the $9 \mathrm{AA}$ and the matrix is the mechanism for the $\mathrm{HB}$ spectrum of neutral 9AA, it is surprising that monoprotonated 9AA is the dominant photoproduct of neutral 9AA at $\mathrm{pH} 13$ since the $\mathrm{p} K_{\mathrm{a}}$ of $9 \mathrm{AA}$ is 9.9 [13]. Furthermore, we suspect that the weaker satellite holes at 514, 650 and $690 \mathrm{~cm}^{-1}$ in the spectrum of neutral 9AA [10] are due to the satellite holes of photoproduct. More interestingly, distinct holes were also observed in the system of 9AA doped in polyvinyl butyral (PVB) film, even though there is no acidic proton in the matrix [14]. In this work, we present a new approach to distinguish the satellite holes between photoreactant and photoproduct. In addition, antihole recovery provides more dynamic information of the excited state of 9AA. Finally, a comparison of the HB mechanisms between 9AA/Gl: $\mathrm{H}_{2} \mathrm{O}$ and 9AA/PVB is discussed.

\section{Experimental}

The experimental setup of the HB method was described elsewhere [15]. The absorption spectrum was obtained by using a home-made double beam spectrometer with a resolution of $\approx 0.03 \mathrm{~nm}$. An intracavity frequency-doubled optical parametric oscillator (OPO) pumped by a $Q$-switched and modelocked Nd:YAG laser (Quantronix 416) was constructed in our laboratory to serve as a burning light source [9]. The laser emitted trains of $\approx 10$ pulses separated by $13 \mathrm{~ns}$ at a repetition rate of $500 \mathrm{~Hz}$. The output wavelength of the OPO system can be tuned from 380 to $480 \mathrm{~nm}$ with a bandwidth less than 2 $\mathrm{cm}^{-1}$.

The 9AA molecule was purchased from Acros and used without further purification. The sample of 9AA doped in glycerol-water glass was prepared from a saturated aqueous solution of $9 \mathrm{AA}$ by diluting it to $1 / 50$ with glycerol:water solution $(5: 4 \mathrm{v} / \mathrm{v})$ at $\mathrm{pH} 13$. The $\approx 1$ magnitude of the optical density at $435 \mathrm{~nm}$ absorption band corresponds to the concentration of $\approx 5 \times 10^{-4} \mathrm{M}$. Solutions were introduced into a quartz tube with an inner diameter of $1.5 \mathrm{~mm}$ and placed in a brass holder with several drill holes of $\approx 1 \mathrm{~mm}$ diameter. Clear glasses were normally formed by introducing the sample directly into a Janis dewar from room temperature to $6 \mathrm{~K}$.
The sample of 9AA/PVB film was prepared by dissolving the 9AA and the PVB powder (Polysciences, Inc.) in organic solvent of $\mathrm{CH}_{2} \mathrm{Cl}_{2}$. The syrupy solutions were poured onto a glass plate and then placed into a desiccator to dry into a film of thickness $\approx 70 \mu \mathrm{m}$.

\section{Results and discussion}

The dashed line and dotted line in Fig. la show the absorption spectra of 9AA doped in $\mathrm{Gl}: \mathrm{H}_{2} \mathrm{O}$ glasses with $\mathrm{pH}=7$ and 13 , respectively. Our previous study indicated that the monoprotonated 9AA is predominant at $\mathrm{pH} 7$ and the imino form of neutral 9AA is dominant at $\mathrm{pH} 13$ [10]. The lowest absorption band of the monoprotonated 9AA is located at $\approx 425 \mathrm{~nm}$, whereas that of the neutral 9AA is located at $\approx 435 \mathrm{~nm}$. The solid line in Fig. 1a shows the absorption spectrum of neutral 9AA doped in $\mathrm{Gl}: \mathrm{H}_{2} \mathrm{O}$ glass after three consecutive burnings at 437

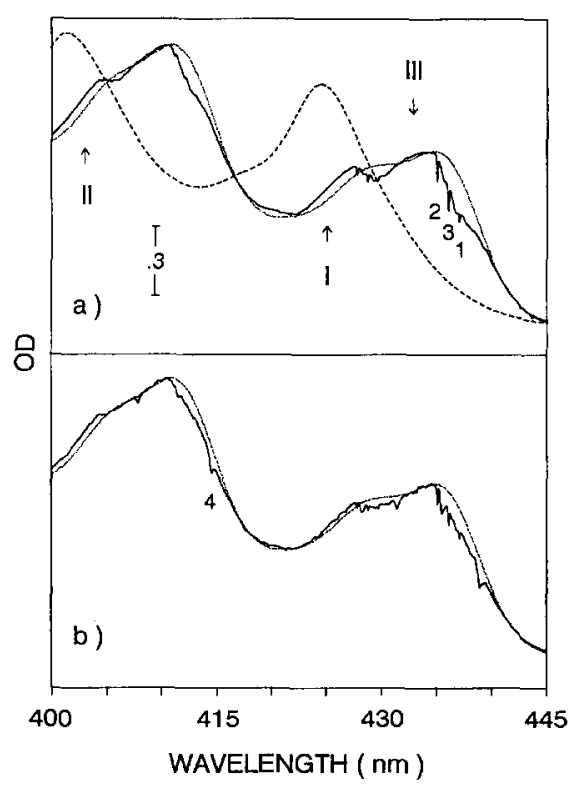

Fig. 1. (a) Absorption spectra of the 9AA doped Gly $-\mathrm{H}_{2} \mathrm{O}$ glass with $\mathrm{pH}=7$ (dashed line) and $\mathrm{pH}=13$ (dotted line). Solid line shows the postburned absorption spectrum of the $\mathrm{pH}=13$ glass with consecutive burning at 435,437 and $436 \mathrm{~nm}$. (b) Solid line is the postburn absorption spectrum after additional $1 \mathrm{~h}$ burning at $415 \mathrm{~nm}$. The numerical number presents the burning order and the arrow indicates the location of antihole. 
(hole 1), 435 (hole 2) and $436 \mathrm{~nm}$ (hole 3) with the pulse energies of $\approx 2.5,3$ and $4 \mu \mathrm{J}$ and the burning time of $60 \mathrm{~min}$ for each excitation, respectively. The most important features presented in Fig. 1a are the antiholes in the region of $420-430 \mathrm{~nm}$ (antihole I) and the blue-side of the $405 \mathrm{~nm}$ (antihole II). These are consistent with the absorption bands of the monoprotonated 9AA. It is noted that antiholes I and II in Fig. 1 a continue growing during the consecutive excitations. We further examine whether the satellite holes of photoproduct can be produced.

The solid line in Fig. 1b shows the further absorption spectrum of the solid line in Fig. 1a after burning at $415 \mathrm{~nm}$ with the pulse energy of $1.5 \mu \mathrm{J}$ and the burning time of $60 \mathrm{~min}$. The difference between the two solid lines in Fig. 1a and $1 b$ gives the HB spectrum as shown in Fig. 2a. For reference, Fig. $2 b$ and the inset in Fig. $2 b$ show the HB spectra of neutral 9AA and monoprotonated 9AA at $\approx 415$ $\mathrm{nm}$, respectively. A comparison between the satellite holes in Fig. $2 \mathrm{a}$ and Fig. $2 \mathrm{~b}$ shows that the $514 \mathrm{~cm}^{-1}$ is a new satellite hole. In addition, the satellite holes at 650 and $690 \mathrm{~cm}^{-1}$ become stronger relative to the $555 \mathrm{~cm}^{-1}$ satellite hole. It is found that the 514,650

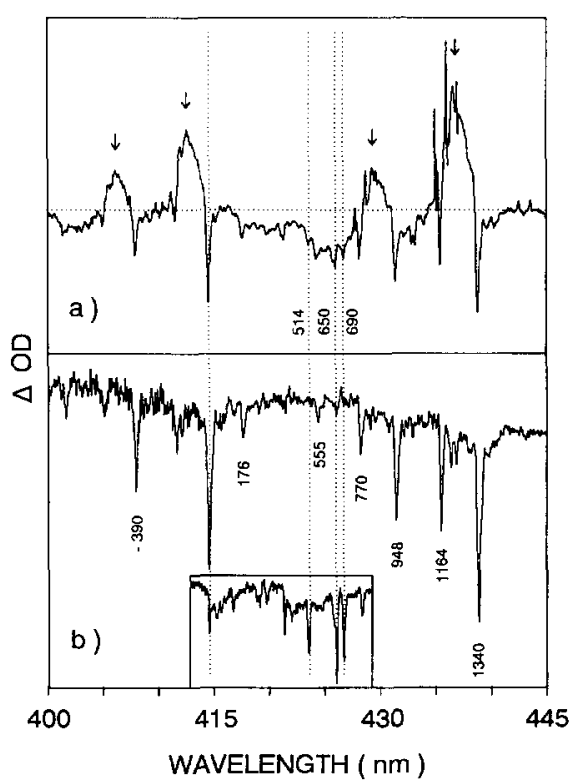

Fig. 2. (a) The HB spectrum obtained from the difference between the two solid lines in Figs. 1a and 1b. (b) The HB spectra of neutral 9AA and monoprotonated 9AA (insert) after burning $1 \mathrm{~h}$ at $415 \mathrm{~nm}$. and $690 \mathrm{~cm}^{-1}$ satellite holes are prominent in the HB spectrum of monoprotonated 9AA as shown in the inset of Fig. 2b. In our previous work [10], the $514 \mathrm{~cm}^{-1}$ satellite hole was assigned to the $\mathrm{NH}_{2}$ vibration of monoprotonated $9 \mathrm{AA}$ and the $555 \mathrm{~cm}^{-1}$ satellite hole was attributed to a difference of the overtone of the $390 \mathrm{~cm}^{-1}$ mode and the $1338 \mathrm{~cm}^{-1}$ mode of neutral 9AA. Apparently, Fig. 2a contains not only the satellite holes of neutral 9AA but also the satellite holes of photoproducts. Although the $1368 \mathrm{~cm}^{-1} \mathrm{SH}$ is difficult to justify because of the interference from the tail of the strong $\mathrm{SH}$ at 1338 $\mathrm{cm}^{-1}$, we believe that the 514, 650 and $690 \mathrm{~cm}^{-1}$ holes in Fig. 2a are the satellite holes of photoproduct. In summary, these distinct satellite holes confirm that monoprotonated 9AA is the photoproduct of neutral 9AA in $\mathrm{Gl}: \mathrm{H}_{2} \mathrm{O}$ at $\mathrm{pH} 13$.

INDO calculations [16] indicated that the electron density of neutral 9AA migrates from the endocyclic nitrogen atom to the carbon atom in the short axis and the exocyclic nitrogen atom during excitation. As a result, the exocyclic nitrogen becomes more basic, while the endocyclic nitrogen becomes more acidic. Consequently, a photoinduced proton transfer occurs from solvent to the exocyclic nitrogen of 9AA, and another photoinduced proton transfer takes place from the endocyclic nitrogen of 9AA to solvent. Therefore, Chiang et al. [10] proposed that the intermolecular proton transfer between the chromophore and matrix, triggered by electron migration along the short axis of 9AA via electronic excitation, is the main mechanism for the hole formation.

The hydrogen bonding between the 9AA and the neighboring matrix would alter the environmental configurations after electronic excitation. As a result, spectral holes can be also produced. A weak blueshifted antihole III near the ZPH is observed in Fig. 1a. The possible mechanism for the blue-shifted antihole of the ZPH was discussed by Shu and Small [17]. They suggested that nonphotochemical hole burning (NPHB) occurs as a result of an outside-in hierarchy of tunneling events, triggered by electronic excitation. Consequently, the free volume for the chromophore increases for the postburn configuration. The increase in free volume can explain the blue-shifted antihole since $\pi \pi^{*}$ transitions typically undergo a red-shift from gas to condensed phases. We believe that the $\mathrm{HB}$ mechanism of $9 \mathrm{AA} / \mathrm{Gl}: \mathrm{H}_{2} \mathrm{O}$ 
consists of both photochemical hole burning and NPHB processes. However, the relative intensities between two antiholes resulted from these two mechanisms suggest that photochemical hole burning is much more dominant.

The HB spectrum of 9AA/PVB film has been studied by Bogner et al. [14] for the application of optical data storage. In the system of 9AA/PVB, no proton is available for proton transfer between the chromophore and the PVB film matrix. The distinct holes observed in their HB spectra implies that proton transfer between the 9AA and the matrix is not necessary for the hole formation in the spectrum of 9AA/PVB. In order to examine the HB mechanism of 9AA, we have further studied the satellite holes and antiholes of 9AA doped in PVB film.

Fig. 3a shows the preburn and postburn absorption spectra of 9AA doped in PVB film taken at $\lambda_{\mathrm{B}}=439 \mathrm{~nm}$. The pulse energy is $\approx 2.5 \mu \mathrm{J}$ and the burning time is $200 \mathrm{~min}$. The antiholes, barely observed in Fig. 3a, are different from the antiholes observed in Fig. la. In addition, the $\approx 7 \%$ hole depth of the saturated $\mathrm{ZPH}$ at $439 \mathrm{~nm}$ in the spectrum of $9 \mathrm{AA} / \mathrm{PVB}$ is much shallower than the $\approx$ $35 \%$ hole depth of the saturated $\mathrm{ZPH}$ at $437 \mathrm{~nm}$ in

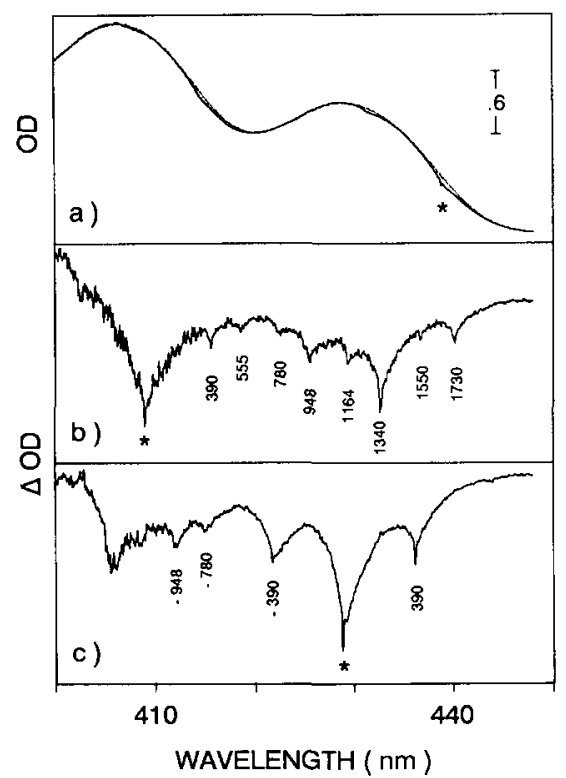

Fig. 3. Preburn and postburn absorption spectra of the 9AA doped in PVB film taken at $\lambda_{B}=439 \mathrm{~nm}$ (a), HB spectra at $409 \mathrm{~nm}$ (b) and at $429 \mathrm{~nm}(\mathrm{c})$. the spectrum of 9AA/Gl: $\mathrm{H}_{2} \mathrm{O}$ [10]. Fig. $3 \mathrm{~b}$ and $3 \mathrm{c}$ show the HB spectra of 9AA/PVB taken at $\lambda_{\mathrm{B}}=409$ and $429 \mathrm{~nm}$, respectively. The pulse energies are $\approx 1.5 \mu \mathrm{J}$ and $2.2 \mu \mathrm{J}$ and the burning time are 80 and $140 \mathrm{~min}$, respectively. It is found that the satellite holes observed in Fig. $3 \mathrm{~b}$ are quite similar to that in Fig. 2b. The high resolution of satellite holes is useful for characterizing the tautomer structures of 9AA. According to our previous study of the satellite holes of 9AA [10], the appearance of the $1340 \mathrm{~cm}^{-1}$ satellite hole and the absence of $514 \mathrm{~cm}^{-1}$ satellite hole in Fig. 3 suggest that the imino form of neutral 9AA is the predominant structure in PVB film.

Considering that electron migrates from the endocyclic nitrogen to the exocyclic nitrogen upon photoexcitation [16], photoinduced intramolecular proton transfer from the endocyclic nitrogen atom to the exocyclic nitrogen atom may be responsible for the 9AA/PVB system. However, the distance between endocyclic and exocyclic nitrogen atoms is estimated to be $\approx 4 \AA[18]$, the imino-amino transformation mechanism is not very favorable. Since the low quantum yield of HB signal can be accumulated, one cannot completely eliminate the possibility of the photoinduced intramolecular proton transfer. Another possible mechanism for the $\mathrm{HB}$ mechanism of 9AA/PVB is the NPHB. The absence of the blueshifted antihole near the ZPH in Fig. 3 may be attributed to the low efficiency of hole formation and the extreme width characteristic of antiholes [19]. In addition, larger electron-phonon coupling can make the justification of the blue-shifted antihole near the $\mathrm{ZPH}$ difficult. The Debye-Waller factor measured in the spectrum of 9AA/PVB is 0.2 [20], while the Debye-Waller factor determined by the short time limit HB method for the $9 \mathrm{AA} / \mathrm{Gl}: \mathrm{H}_{2} \mathrm{O}$ at $\mathrm{pH} 13$ is $\approx 0.63$. Note that the electronic interactions of the 9AA with the PVB can alter the environmental configurations. Consequently, spectral holes are produced. The lower hole burning efficiency and higher electron-phonon coupling observed in the spectrum of the imino form of neutral 9AA doped in PVB relative to that in $\mathrm{Gl}: \mathrm{H}_{2} \mathrm{O}$ suggest that the change of environmental configurations may be one of the mechanism for producing the holes in the spectrum of 9AA/PVB film. More experiments are necessary to determine the HB mechanism of 9AA/PVB. Nevertheless, the main mechanism for the imino form of 
neutral 9AA doped in Gl: $\mathrm{H}_{2} \mathrm{O}$ glass and in PVB film is quite different according to their $\mathrm{HB}$ spectra.

Another noteworthy feature in Fig. $2 \mathrm{a}$ is the presence of antiholes. These antiholes are apparently due to laser induced hole filling (LIHF) of the primary holes. The solid-line spectrum in Fig. $1 \mathrm{~b}$ indicates that there are still holes relative to the original absorption spectrum in the regions labeled as antiholes in Fig. 2a. The phenomena of LIHF have been studied by several group [21-27]. Possible mechanisms of LIHF involve local heating [22], spectral diffusion [23] and reversion resulting from antihole excitation $[22,24,25]$. In our work, the lack of appreciable line broadening in LIHF allows us to eliminate spectral diffusion. This linewidth behavior has been observed in several other LIHF studies [26]. In the study of a binary mixture of ACMA and BODIPY doped in $\mathrm{Gl}: \mathrm{H}_{2} \mathrm{O}$ glass [27], Kuo and Chang found that the primary holes of ACMA burned at $428 \mathrm{~nm}$ were not filled by the secondary excitation of the absorption band of BODIPY at $555 \mathrm{~nm}$. Similar results were also observed in the study of a mixture of 9AA and BODIPY doped in $\mathrm{Gl}: \mathrm{H}_{2} \mathrm{O}$ glass. Therefore, local heating is an unlikely mechanism for the hole filling. Shu and Small [25] studied the LIHF of cresyl violet doped in PVOH films and found that hole filling is due to light-induced antihole reversion. They concluded that the dominant mechanism involves electronic excitation of the antihole sites by means of their broad phonon sideband. Olson et al. [22] found that reversion resulting from excitation of primary antihole is the mechanism of LIHF in the system of pentacence doped in benzoic acid. They further suggested that hydrogen bond tautomerization of the host is involved in the hole burning mechanism. Furthermore, antihole recovery of the holes is consistent with the tautomerization mechanism. The photoinduced tautomer is not the most stable form, excitation of the antihole allows the photoinduced tautomer to go back to the stable form.

In this work, 9AA exhibits lower basicity in the first excited state than in the ground state [16]. The excited state $\mathrm{p} K_{\mathrm{a}}^{*}$ of $9 \mathrm{AA}$ was estimated to be lower than $\mathrm{p} K_{\mathrm{a}}$ by at most $0.6 \mathrm{pH}$ units by Smith et al. [28]. Since the excited state $\mathrm{p} K_{\mathrm{a}}^{*}$ of $9 \mathrm{AA}$ is $\approx 9.3$, the excited state 9AA is not very favored to be protonated at $\mathrm{pH} 13$. Only minor yield is expected, the small quantum yield of the photoproduct is a result of strong competition of electronic relaxation from $S_{1}$ versus the unfavorable proton transfer processes. In the HB spectra, antihole recovery in Fig. $1 b$ and new antiholes in Fig. 2a resulting from reversion of the photoproduct suggest that the excited state 9AA returns to the ground state imino form of neutral 9AA predominantly. It is reasonable because the imino form of neutral 9AA is more stable than the monoprotonated 9AA at $\mathrm{pH} 13$. Reversion of photoproduct back to the original state has been observed in the photochemistry of chlorin in crystalline n-alkanes [11]. However, the photoinduced antiholes I and II in Fig. 1a remain growing during the consecutive excitations at 437,435 and $436 \mathrm{~nm}$ (not shown), indicating that part of the excited state 9AA can go to the monoprotonated 9AA. The mechanism of photoinduced reaction of neutral 9AA doped in $\mathrm{Gl}: \mathrm{H}_{2} \mathrm{O}$ glass is summarized in Fig. 4. The advantage of $\mathrm{HB}$ method is that the HB signal of low quantum yield photoproduct can be accumulated to produce high resolution spectral holes.

In summary, we have demonstrated that the satellite holes of the photoproduct can be used to characterize the molecular structure of the photoproduct
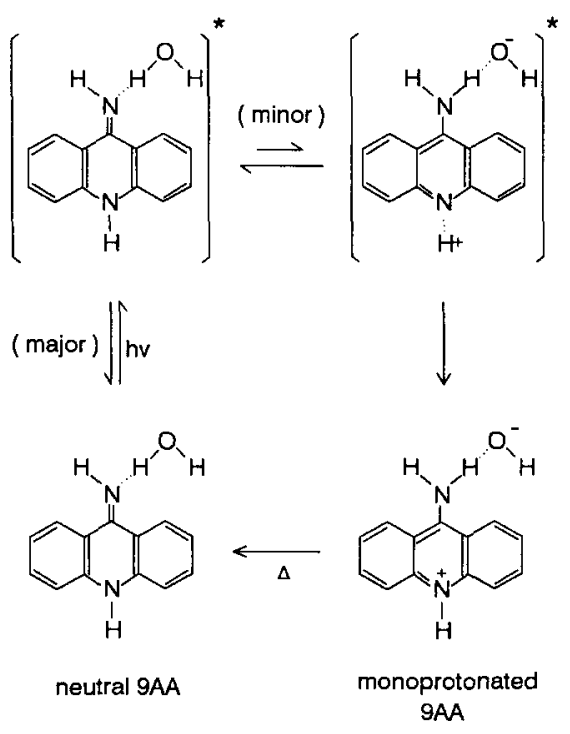

Fig. 4. Schematic diagram of proposed photoreaction for the imino form of neutral 9AA doped in $\mathrm{Gl}: \mathrm{H}_{2} \mathrm{O}$ at $\mathrm{pH} 13$. The solid line presents a chemical bond and the dash line denotes a hydrogen bond. The triangle delineates the increase of temperature. 
and determine the HB mechanism. In addition, the antihole recovery provides additional information about the photoinduced reaction. Although the excited state of 9AA returns directly to the imino form of neutral 9AA at $\mathrm{pH} 13$ predominantly, the low quantum yield photoproduct of the monoprotonated 9AA can be accumulated and clearly identified by the satellite holes in the HB spectrum. It is clear that satellite holes and antiholes can be used to determine the molecular structures of photoproducts and to probe the photoinduced reaction. Furthermore, the $\mathrm{HB}$ mechanism of the imino form of neutral 9AA doped in $\mathrm{Gl}: \mathrm{H}_{2} \mathrm{O}$ glass is mainly due to intermolecular proton transfer between 9AA and matrix. However, the spectral holes in the spectrum of $9 \mathrm{AA} / \mathrm{PVB}$ film is tentatively ascribed to the change of environmental configurations. The investigation of the distinct satellite holes and antiholes is important for the study of the interactions of 9AA with DNA [29], which is under progress in our laboratory.

\section{Acknowledgements}

This work was supported by the Academia Sinica and the NSC of the Republic of China (Grant No. NSC-86-2113-M001-045).

\section{References}

[1] W.E. Moerner (Ed.), Topics in Current Physics 44, Persistent Spectral Hole Burning: Science and Applications, Springer, Berlin, 1988.

[2] J. Friedrich, D. Haarer, Angew. Chem. Int. Ed. Engl. 23 (1984) 113.

[3] S. Volker in: Relaxation Processes in Molecular Excited States, J. Funfschilling (Ed.) Kluwer, Dordrecht, 1989; Ann. Rev. Phys. Chem. 40 (1989) 499.
[4] L.R. Narasimhan, K.A. Littau, D.W. Pack, Y.S. Bai, A. Elschner, M.D. Fayer, Chem. Rev. 90 (1990) 439.

[5] R. Jankowiak, J.M. Hays, G.J. Small, Chem. Rev. 93 (1993) 1471.

[6] J. Friedrich, D. Haarer, J. Chem. Phys. 79 (1983) 1612.

[7] R.A. Avarmaa, K.K. Rebane, Spectrochim. Acta A 41 (1985) 1365.

[8] J.K. Gillie, G.J. Small, J.H. Golbeck, J. Phys. Chem. 93 (1989) 1620.

[9] T.-C. Chang, C.C. Chiang, J.Y. Cheng, Trends Chem. Phys. 4 (1996) 141.

[10] C.C. Chiang, B.C. Hwang, J. Yu, J.Y. Cheng, C.Y. Mou, S.H. Lin, T.-C. Chang, J. Chem. Soc. Faraday Trans. 93 (1997) 1297.

[11] S. Volker, R.M. Macfarlane, IBM J. Res. Dev. 23 (1979) 547; J. Chem. Phys. 73 (1980) 4476.

[12] C.C. Chiang, J.Y. Cheng, Y.R. Cheng, H.S. Chen, C.Y. Mou, T.-C. Chang, Mol. Cryst. Liq. Cryst. 291 (1996) 175.

[13] R.M. Acheson, Acridines, Interscience, New York, 1956.

[14] U. Bogner, K. Beck, M. Maier, Appl. Phys. Lett. 46 (1985) 534.

[15] T.-C. Chang, C.C. Chiang, G.J. Small, S.H. Chou, Chem. Phys. Lett. 223 (1994) 190.

[16] J. Rak, J. Blazejowski, J. Photochem. Photobiol. A 67 (1992) 287.

[17] L. Shu, G.J. Small, J. Opt. Soc. Am. B 9 (1992) 724.

[18] R. Talacki, H.L. Carrell, J.P. Glusker, Acta. Crystallogr. B 30 (1974) 1044.

[19] Personal communication, referee.

[20] I. Renge, J. Opt. Soc. Am. B 9 (1992) 719.

[21] J.M. Hayes, G.J. Small, Chem. Phys. 27 (1978) 151.

[22] R.W. Olson, H.W.H. Lee, F.G. Patterson, M.D. Fayer, R.M. Shelby, D.P. Burum, R.M. Macfarlane, J. Chem. Phys. 77 (1982) 2283.

[23] W. Kohler, J. Meiler, J. Friedrich, Phys. Rev. B 35 (1987) 4031.

[24] R. van den Berg, S. Volker, Chem. Phys. 128 (1988) 257.

[25] L. Shu, G.J. Small, J. Opt. Soc. Am. B 9 (1992) 738.

[26] N. Murase, K. Horie, Chem. Phys. Lett. 209 (1992) 42.

[27] C.T. Kuo, T.-C. Chang, J. Chem. Phys. 106 (1997) in press.

[28] C.A. Smith, H.C. Chang, W.S. Struve, G.J. Atwell, W.A. Denny, J. Phys. Chem. 99 (1995) 8927.

[29] J.Y. Cheng, C.C. Chiang, Y.R. Cheng, H.S. Chen, L.J. Lin, T.-C. Chang, J. Chin. Chem. Soc. 44 (1997) in press. 